\title{
LA REFORMA MILITAR DE LOS GOBIERNOS DE SUAREZ*
}

Agustín Rodríguez Sahagún

La existencia, hasta la segunda mitad de 1977, de tres Ministerios diferentes - uno para cada Ejército- había generado una estructura dispersa y heterogénea de las Fuerzas Armadas, con abundancia de mandos y una gran diversidad de escalas y servicios, con unos recursos bumanos excesivamente numerosos, pero insuficientemente dotados de armamento y material, y con una distribución de la fuerza en la que pesaban más la inercia o la atención a la problemática interior que la visión global de las necesidades de defensa de nuestras fronteras frente al eventual enemigo exterior. Todo ello, a pesar de los esfuerzos del Alto Estado Mayor que intentaba una adecuada coordinación sin conseguirla, por falta del marco legal necesario y la inexistencia de un mando único. Esta situación incidía negativamente en la operatividad de la fuerza disponible y en la posibilidad de sacar el máximo provecho a los recursos de la Defensa, y había llegado a producir un alto nivel de dependencia estratégica del exterior en los suministros y el mantenimiento de material, así como importantes deficiencias asistenciales de los funcionarios militares, en comparación con otros estamentos de la Administración Civil del Estado.

La llamada reforma militar — que comenzó el general Gutiérrez Mellado cuando era ministro de Defensa, y yo me ocupé de proseguir durante los años

* Publicado en «Historia de la transición», $2 .^{2}$ parte, pp. 681 y ss., editada por Diario 16, suplemento dominical del núm. 152, del 19 de agosto de 1984.

\section{RếS}


que estuve al frente de dicho Departamento- trataba de poner fin a esta situación, mediante la integración de los tres Ejércitos, con el objetivo de disponer de las Fuerzas Armadas que precisa un Estado moderno y democrático para cumplir - a las órdenes del Gobierno de la nación- las misiones que les asigna el artículo $8 .^{\circ}$ de nuestra Constitución. Unas Fuerzas Armadas permanentemente a punto, capaces en sí mismas de ser un factor de disuasión - porque importa más evitar una guerra que ganarla-; con posibilidad de reacción inmediata ante cualquier agresión exterior - porque es preciso disponer de los medios necesarios para defender los intereses españoles en caso de conflicto-, y con capacidad de respaldo de nuestra politica internacional, ya que en el mundo en que vivimos sólo se puede tener la autonomía de decisión imprescindible si se cuenta con los medios en que apoyarla.

Gutiérrez Mellado sentó las bases para conseguir esta integración al crear la Junta de Jefes de Estado Mayor (JUJEM), como órgano colegiado superior de la cadena de mando militar, responsable de la máxima eficacia operativa conjunta de los tres Ejércitos, y al estructurar, en la segunda mitad de 1977, el Ministerio de Defensa. La creación del nuevo Ministerio se hizo agrupando las estructuras preexistentes de los Ministerios anteriores y estableciendo una serie de órganos comunes para los tres Ejércitos, desde una perspectiva global, con el fin de conseguir la necesaria integración en un proceso gradual de adaptación y cada vez mayor coordinación (recibieron especial apoyo, entre otros, la Dirección de Armamento y Material, que ayudó a coordinar las compras, a realizar y sacar el mejor partido posible de ellas, y el Centro Superior de Información de la Defensa, que permitió avanzar de forma importante en la profesionalización y potenciación de los Servicios de Inteligencia). Sabíamos que el proceso tenía que ser necesariamente largo para rendir plenos frutos, dado lo que habían tardado en consolidarse experiencias semejantes en otros países occidentales, pero se avanzó mucho desde el verano de 1977 hasta principios de 1981. De la complejidad de la reorganización emprendida da idea el que afectaba directamente a más de 400.000 personas -unos 100.000 militares profesionales, 40.000 funcionarios civiles y unos 300.000 soldados de reemplazo-, además de a las clases pasivas correspondientes. De ahí los continuos esfuerzos que tuvimos que hacer para reducir el nivel de burocratización existente en sus estructuras. Así, por ejemplo, recuerdo que, a poco de ocupar mi cargo, tuve que tomar medidas para que los familiares de las víctimas del terrorismo pudieran cobrar de inmediato la pensión a que tenían derecho, que, a veces, se demoraba meses y meses como consecuencia de los trámites que requerían las formalidades de la burocracia, añadiendo con este alargamiento de los plazos serias dificultades económicas a la ya trágica desgracia que sufrían. Combatir la burocratización exigió cambios orgánicos, pero sobre todo aplicar un esfuerzo sistemático de organización con el empleo de nuevos métodos administrativos y de gestión, y la incorporación de las téc- 
nicas informáticas al proceso de planificación y decisión, con lo que se consiguió un avance sustancial para aumentar la rentabilidad de los recursos económicos, y se pudo presentar, ya en 1980 , el proyecto de Presupuesto de Defensa por Programas.

En esa misma línea - y una vez dispuestas las estructuras adecuadas de coordinación - se comenzó a desarrollar, con el denominado Ciclo de Defensa, una nueva Política de Defensa que, además de incorporar los sistemas más modernos de planteamiento, programación y presupuesto, ponía el acento en la determinación y distribución geográfica de la estructura de fuerza precisa para atender las necesidades de defensa exterior (con la consiguiente modificación de las regiones militares existentes, a través del META, y restantes planes de modernización). En los años 79/80 se elaboró, por primera vez, un «Plan Estratégico Conjunto» (PEC), que incluía los objetivos estratégicos a alcanzar, la evaluación de las amenazas posibles, la propuesta de estrategia conjunta y el objetivo de fuerza, que se consideraba necesario para la década de los ocbenta, y que hubo de revisarse a fin de ajustar el binomio «necesidades de la defensa-posibilidades de la nación». Todos estos trabajos culminarían con el proyecto de la nueva ley de dotaciones para la modernización de las Fuerzas Armadas. Además del gran esfuerzo que realizaron los entonces miembros de la JUJEM - tenientes generales Ignacio y Emiliano Alfaro Arregui, teniente general Gabeiras y almirante Arévalo- colaboraron también decisivamente en este proceso el almirante Liberal como subsecretario de Defensa, el general Munilla como secretario de Política de Defensa y el almirante Díaz Deus primero, y el general Lacalle después, como secretarios de Asuntos Económicos.

La planificación a medio y largo plazo que entrañaba el PEC permitió desarrollar una política de apoyo a la industria nacional de armamento, con el objetivo de aumentar el nivel de abastecimiento propio en las dotaciones de nuestros Ejércitos y disminuir la exagerada dependencia estratégica del exterior, al tiempo que se ayudaba a la industria española a superar la crisis existente. A tal fin, de una parte, se dieron instrucciones para que las compras de armamento que se realizaban al extranjero se aprovecharan no sólo para obtener contrapartidas comerciales e industriales, sino también contrapartidas tecnológicas que permitieran iniciar nuevas fabricaciones (con un énfasis especial en el campo de la electrónica) y, de otra, se iniciaron contactos y se firmaron acuerdos con diversos paises europeos (Alemania, Italia, Francia, Gran Bretaña...) con la mira puesta no sólo en la realización de cofabricaciones, sino sobre todo en la participación en proyectos conjuntos, que permitieran apoyar nuestra investigación y estar presentes en el desarrollo $y$ fabricación de los sistemas europeos de armas del futuro. Todo ello impulsó fuertemente nuestras exportaciones, al tiempo que el mayor indice de integración nacional del armamento conseguido nos evitaba algunas de las importa- 
ciones que antes hacíamos y permitía sacar más provecho de los recursos económicos disponibles.

En el plano legislativo hay algunas normas que me parecen necesario destacar. En primer lugar, las nuevas Reales Ordenanzas de Juan Carlos I, auténtico código moral de los derechos y deberes del militar en todos sus grados, que representaron un importantísimo trabajo de renovación y actualización de las Ordenanzas de Carlos III en lo que se refiere a la parte común de los tres Ejércitos, y fueron aprobados a finales de 1978, siendo ministro de Defensa el general Gutiérrez Mellado. La comisión creada al efecto, e integrada por un escogido grupo de generales, jefes y oficiales de los tres Ejércitos, continuó posteriormente sus trabajos con la elaboración del desarrollo específico de las ordenanzas de cada uno de ellos, cuyo proyecto se presentó a finales de 1980 .

En la primera mitad de ese año aprobaban el Congreso y el Senado la ley orgánica de criterios básicos de la Defensa Nacional y la Organización Militar, que en desarrollo de lo preceptuado en la Constitución regulaba las estructuras y competencias de los diversos órganos politicos $y$ militares que participan en la Defensa Nacional, así como los criterios básicos de su organización y funcionamiento. La ley fijaba con claridad la dependencia de las Fuerzas Armadas del Gobierno - la llamada supremacia del poder civil- al establecer, en su artículo 11, que la Junta de Jefes de Estado Mayor, órgano colegiado superior de la cadena de mando militar, depende del presidente de Gobierno, quien ejerce su autoridad a través del ministro de Defensa, salvo en aquellas materias que se reserve expresamente para sí. Por su parte, el artículo 12 señalaba que los jefes de los Estados Mayores del Ejército de Tierra, de la Armada y del Ejército del Aire ejercen el mando militar de sus respectivos Ejércitos, bajo la autoridad del ministro de Defensa, mientras que el artículo 7 de la misma ley establecía que el Gobierno determina la política de defensa y, por tanto, la política militar que forma parte de aquella. Asimismo, esta norma orgánica perfilaba en su texto una serie de leyes ordinarias que desarrollarían los criterios básicos y que completaban los diversos aspectos de la reforma en lo referente a la movilización, a la organización de cada uno de los Ejércitos, la enseñanza militar, etc. No quiero dejar de referirme - por la importancia que creo representaba como símbolo- a la nueva fórmula constitucional de juramento de la bandera, aprobada también en 1980, acomodando éste a lo establecido en nuestra norma fundamental.

En cuanto a la reforma de la justicia militar se optó por hacerla en dos fases. La primera, referente a los aspectos esenciales, del código, tenía como objetivos esenciales el fortalecimiento de las garantías procesales y la limitación de la jurisdicción militar al ámbito estrictamente castrense, adecuando la normativa a lo preceptuado por la Constitución y evitando la conflictividad que se venía suscitando por la actuación de la autoridad militar frente a civi- 
les en materias ajenas a la misión y función de las Fuerzas Armadas, como libertad de expresión, etc. Esta reforma parcial, aprobada en 1980, fue saludada como un importante avance por los portavoces de todas las fuerzas parlamentarias. En ella se comprometía el envío a las Cortes, en el plazo de un año, de un nuexo texto completo del Código de Justicia Militar que sustituyera en su integridad al vigente y estuviera debidamente coordinado con los planteamientos del nuevo Código Penal. Para su elaboración se creó una comisión presidida por el director de la Escuela de Estudios Jurídicos, que recibió como líneas directrices de sus trabajos:

- La más estricta adecuación a la letra y el espíritu de la Constitución y su coordinación en el nuevo Código Penal.

- La elaboración de la nueva normativa en varios textos legales (penal, procesal, etc.), de forma que se facilitara su mejor conocimiento y permanente actualización.

- La conveniencia de reservar al código castrense la materia delictiva, relegando todo lo disciplinario al reglamento de disciplina militar, distinguiendo así entre faltas penales y disciplinarias.

La comisión trabajó a fondo y, a principios de 1981, entregaba un primer borrador, pero, sin embargo, ésta es la fecha en que el nuevo código todavía no ha sido aprobado por el Congreso de los Diputados.

Especial atención nos mereció el desarrollo de una cuidadosa política de personal, que permitiera situar al bombre adecuado en el puesto adecuado, mediante la acomodación de las aptitudes a las necesidades e importancia de la función a desempeñar. Tratando de hacer siempre las cosas desde la comprensión y el afecto a las Fuerzas Armadas como institución y desde el respeto a sus miembros, pero sin renunciar tampoco en ningún momento a las facultades que correspondian al Gobierno, ya fuera a la hora de establecer los ascensos o de seleccionar atentamente los destinos, o a la de resolver con objetividad los problemas que, a ese respecto, surgían a veces entre los distintos Ejércitos, Cuerpos o Armas.

En esa línea, además de numerosas normas para equilibrar en lo posible los ascensos entre las diversas Armas, reducir sensiblemente el número de soldados de reemplazo y dar facilidades en el cumplimiento del servicio militar, en tanto se elabora la nueva ley, etc., se remitió a las Cortes, en abril de 1980, un importante paquete legislativo que incluía la ley de creación de la reserva activa y fijación de edades de retiro en los tres Ejércitos, la de clasificación de mandos y ascensos para los militares de carrera del Ejército de Tierra y la reorganización de las escalas especial y básicas del mismo Ejército, de gran importancia para los suboficiales. Con estos proyectos se pretendía facilitar la política de personal a medio plazo, al tiempo que conseguir el rejuvenecimiento de nuestros Ejércitos, una mayor eficacia de las 
Fuerzas Armadas y la posibilidad de una mejor realización profesional. Para ver la importancia del rejuvenecimiento a lograr con esta regulación, baste recordar, como anécdota, que con su plena aplicación se podría contar con generales de cincuenta años, en línea con lo que ocurre en otros Ejércitos modernos occidentales.

En materia de personal se hizo también un gran esfuerzo en todo lo que se refería a la acción social, no sólo poniendo en marcha el Instituto Social de las Fuerzas Armadas para prestar atención directamente o mediante conciertos a sus más de 300.000 afiliados, sino mediante una política de apoyos culturales y recreativos, creación de residencias para oficiales y suboficiales, apoyos a las asociaciones de retirados y, sobre todo, un decidido impulso a la construcción de viviendas, que permitió multiplicar en pocos años las viviendas logísticas y en propiedad de que disponían las Fuerzas Armadas, al tiempo que se colaboró así a fomentar la inversión y la creación de puestos de trabajo.

Finalmente, no quiero dejar de referirme a la Enseñanza Militar, aspecto fundamental para los Ejércitos del futuro. En este punto, al tiempo que se eligieron los mejores hombres disponibles para los puestos clave (así, por ejemplo, el general Pinilla para dirigir la Academia General Militar de Zaragoza y el general Cano Hevia al frente de la Escuela de Estado Mayor), además de introducir el conocimiento y enseñanza de la Constitución, se iniciaron una serie de reformas en la formación de los cuadros de mando, que si a veces encontraron dificultad en la inercia de las estructuras, sirvieron para perfilar las bases sobre las que debe asentarse la enseñanza del futuro.

Estas fueron las líneas generales de una reforma necesaria, posible y conveniente, cuya característica principal quizá fue la globalidad de su enfoque, y que contó, desde su inicio, con la decidida voluntad política de los Gobiernos de Adolfo Suárez de llevarla a cabo, y con el aliento y estímulo de S. M. el Rey para la modernización de las Fuerzas Armadas y el mejor cumplimiento de su misión constitucional. 
CRITICA DE LIBROS 